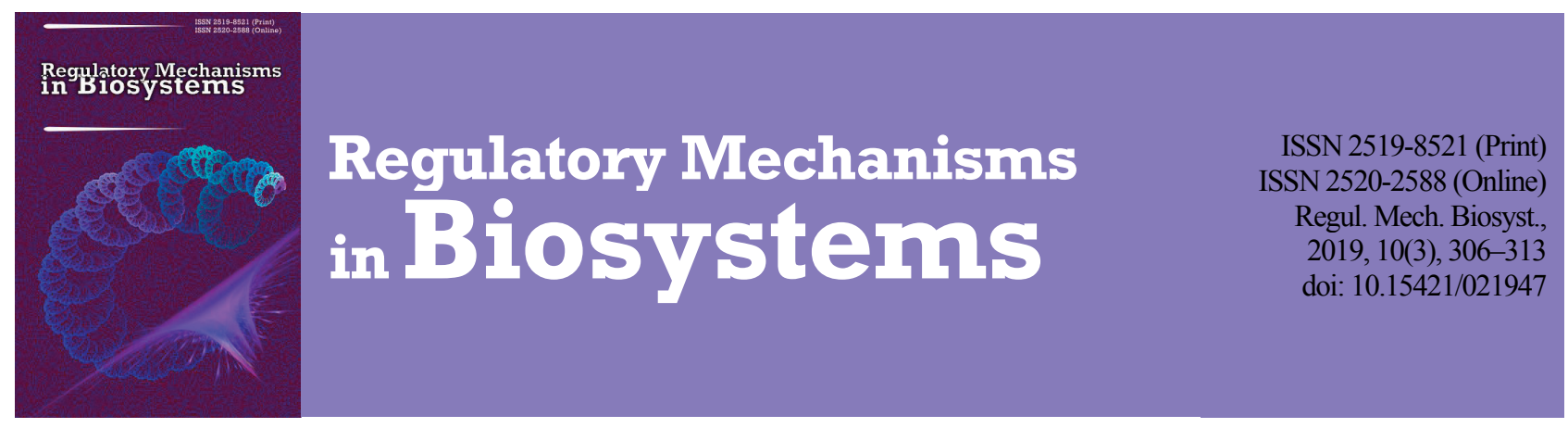

\title{
In silico study of peculiarities of metabolism of erythrocytes with glucosephosphate isomerase deficiency
}

\author{
O. I. Dotsenko
}

\author{
Vasyl Stus Donetsk National University, Vinnytsia, Ukraine
}

Article info

Received 11.06.2019

Received in revised form 16.07.2019

Accepted 17.07.2019

Vasyl Stus Donetsk National University, 600-richya st, 21 ,

Vinnytsia 21021,

Ukraine.

Tel.: +38-093-724-66-10

E-mail:

dots_don@ukr.net

\begin{abstract}
Dotsenko, O. I. (2019). In silico study of peculiarities of metabolism of erythrocytes with glucosephosphate isomerase deficiency. Regulatory Mechanisms in Biosystems, 10(3), 306-313. doi:10.15421/021947
\end{abstract}

\begin{abstract}
Glucose phosphate isomerase (GPI) deficiency, the third most common cause of hereditary nonspherocytic hemolytic anemia, is associated with the mutation of the GPI gene. The results of the GPI deficiency are premature aging of erythrocytes, macrocytosis, reticulocytosis, minor splenomegaly, hyperbilirubinemia and hyperferritinemia, and hemolytic crisis under the influence of exogenous oxidants such as infections or drugs. Regarding the the lack of GPI correction drugs, the theoretical substantiation of supportive therapy based on system biology approaches that would allow the analysis of the relationships between numerical metabolic processes in a cell would be beneficial. The stoichiometric model of erythrocytes' steady state metabolism, including the pathways of Embden-Meyerhof and pentose phosphate (PPP), purine metabolism cycles and glutathione synthesis, has been developed. To predict the redistribution of metabolic flows in erythrocytes under conditions of GPI deficiency, we used the flux balance analysis (FBA). In this approach, calculations of the elementary flux modes (EFMs) and the control-effective flux (CEF) have been performed. Using the CEF evaluation approach, effective profiles of enzymatic reactions depending on the degree of enzyme deficiency were obtained. It has been shown that these relationships can be the basis for future experimental studies. Analysis of the profiles of enzymatic reactions of metabolic networks suggests that erythrocytes are capable of metabolizing other substrates that contribute to overcoming the effects of energy stress in the case of enzymopathies. So, it is shown that erythrocytes can effectively use SAM and adenosine as alternative energy sources. It has been established that the GPI enzymopathy results in a decrease in the flow through the glycolysis and pentose phosphate pathway, resulting in a decrease in the content of such reducing agents as NADPH and GSH, ATP. The processes of the GSH synthesis from amino acids in the cell are shown to be suppressed. Decreased content of NADPH and GSH cause the premature aging of erythrocytes. The target therapeutic approaches that influence the behaviour of the metabolic network of erythrocytes are discussed.
\end{abstract}

Keywords: stoichiometric modeling; elementary flux modes; control-effective flux; system biology; flux balance analysis method; metabolic networks of erythrocytes; enzyme deficiencies.

\section{Introduction}

Hemolytic anemias due to the deficiencies of enzymes involved into energy and redox metabolism in erythrocytes are widespread diseases. Thus, glucose-6-phosphate dehydrogenase deficiency affects at least 400 million people (Cakir et al., 2004; van Zwieten et al., 2014; Chaturvedi et al., 2016; Grace \& Glader, 2018). Deficiencies of other enzymes are less widespread, but also quite common. Despite glucosephosphate isomerase (GPI) deficiencies being quite rare, they are the third frequent cause of nonspherocytic hemolytic anemia (Zaidi et al., 2017; Grace \& Glader, 2018; Burger et al., 2019) associated with the mutation of the GPI gene. This gene contains 18 exons and produces $1.9 \mathrm{~kb}$ mRNA, which encodes a 558 amino acids protein (Lin et al., 2015; Zaidi et al., 2017; Fermo et al., 2019). The molecular characterization of known GPI variants indicates that gene defects are predominantly point mutations (Jamwal et al., 2017; Burger et al., 2019; Fermo et al., 2019). Low enzyme activity and its nonstability results from impaired kinetic properties, reduced thermo stability of the mutant enzyme, or defective protein folding (Kugler \& Lakomek, 2000; Koralkova et al., 2014; Lin et al., 2015).

It is considered that the lack of an enzyme violates the normal functioning of the metabolic pathway which it enters. However, in many cases, the decline in the activity of the enzyme seems to be clearly not enough to disrupt the normal metabolism. In hemolytic anemia associated with enzymopathy, the activity of other key enzymes and the metabolites' content may exceed the normal level up to several times, be- cause of the high reticulocytosis (Rossi et al., 2010, Warang et al., 2012; Mojzikova et al., 2018; Fermo et al., 2019). It is known that young erythrocytes, formed in conditions of intense erythropoiesis, are unstable and age rapidly, especially in the presence of unstable enzymes (Mairbäurl, 2018). From the experimental data it is quite difficult to assess exactly what changes in metabolism are due to the rapid aging of cells. That fact makes it difficult to use biochemical indicators to correct metabolic fluxes to improve the resistance of red blood cells and prolong their life in the bloodstream. For most enzymes, there are no markers that allow a quantitative link to be made between the metabolic disturbance and the viability of the cell. The development of these criteria would allow us to systematize existing data about the associations of enzymopathies with nonspherocytic hemolytic anemia (HNSHA) and to develop tests for the determination of biochemical causes of these pathologies In this regard, computational modeling can play an important role in the systematization of existing data and the understanding of the redistribution of metabolic fluxes in response to a particular stress factor, or a violation of the functions of the enzyme.

Mathematical modeling of metabolic processes can be divided essentially into two distinct conceptual approaches: kinetic and structural modeling (Klipp et al., 2016). Kinetic models require detailed information on the kinetic properties of enzymes and, in the absence of this, often are based on unreliable information about kinetic rate principles, kinetic parameters and the nature of regulation processes (Sajitz-Hermstein \& Nikoloski, 2013). Structural modeling circumvents the problem of 
uncertainties of kinetic information by relying only on structural information. It utilizes reaction stoichiometry and reaction directionalities. The resulting metabolic network then is predominantly examined either by determining particular steady-state flux distributions guided by optimization principles, or by characterizing all elementary flux modes (EFMs) that can support a cell's steady-state (Cakir et al., 2004). Control-effective flux (CEF) analysis is another tool in metabolism assessment (Stelling et al., 2002; Sajitz-Hermstein \& Nikoloski, 2013; Schwartz et al., 2015). The CEFs, which are directly determined from the set of EFMs, represent the importance of each metabolic reaction for an efficient and flexible operation of the entire metabolic network towards the implementation of a cellular objective. For the analysis of enzyme deficiencies in terms of the degree of deficiency, a modification was made on the formulation of CEF calculation (Cakir et al., 2004).

A study using stoichiometry modelling with PGI enzyme deficiency is presented in papers (Cakir et al., 2004; Durmuş Tekir et al., 2006). However, the analysis of these works showed that the individual results of the simulation concerning the PGI enzyme did not coincide with the existing literary data. Also, it has not been established which processes are associated with premature aging of erythrocytes and their hemolytic instability. The aim of this study was to apply the tools of structural metabolic control (EFMs, CEFs in deficiency absence and presence) to study the central metabolism of erythrocytes under the conditions of PGI enzymopathy. To achieve this aim, a stoichiometric model has been developed that includes the main metabolic systems of the erythrocyte: glycolytic and pentose phosphate pathways (PPP), adenylate metabolism, processes of synthesis and transformation of glutathione in the cell. Due to the fact that PGI directly affects the process of glucose uptake in the metabolic pathway, we took into account the possibility of using additional metabolites as energy substrates such as S-adenosylmethionine and adenosine (Komarova et al., 1999; Schuster \& Kenanov, 2005; Sun et al., 2017; Adebiyi et al., 2019). The potential of the presented structural modeling tools for revealing possible sites of metabolic control is analyzed. Study of the redistribution of metabolic fluxes in the case of enzymopathy will help formulate strategies for the further treatment of patients with this pathology.

\section{Material and methods}

The main reactions included in the model are presented in Figure 1 and in Table 1. The developed stoichiometric model includes the following metabolic cycles: the Embden-Meyerhof pathways (r1-r15) and pentose phosphate pathway (r16-r23), purine metabolism cycles (r24-r38) and glutathione synthesis pathway ( $(\mathrm{r} 39-\mathrm{r} 43)$. The model considers the processes essential for the maintenance of erythrocyte viability, namely the reactions to protect the cell against oxidative stress ( $r 44 r 46)$, ATPase processes ( $(\mathrm{r} 47)$, phosphorylation reactions ( $\mathrm{r} 48)$, methemoglobin reduction (r49), and 2,3-diphosphoglycerate binding (D23PG; an important intermediate that modulates hemoglobin-oxygen affinity, $\mathrm{r50}$ ).

The metabolic network is represented by 50 reactions and 52 metabolites, 14 of which are external. External metabolites are glucose, Sadenosylmethionine and adenosine, which are alternative sources of energy. Additionally, the external metabolites are pyruvate and lactate, hypoxanthine, bound form of 2,3-diphosphoglycerate, carbon dioxide, and amino acids that participate in the biosynthesis of glutathione. The stoichiometric model matrix was created in the CellNetAnalyser 2017.

The flux balance analysis (FBA) differs from the kinetic approach in such a way that the result of the modeling is not the change in metabolites concentrations in time, but the picture of the distribution of velocities of metabolic reactions With this approach, the model of metabolism is a system of linear algebraic equations, which are the shortcut from metabolic reactions of the system in the steady state:

$$
\sum_{i=1}^{n} S_{i j} \cdot v_{i}=F_{j}, j=1,2, \ldots, m,
$$

where $\mathrm{n}$ - number of reactions (enzymes) in the cell; $\mathrm{m}$ - number of metabolites in the cell; $S_{i j}$-stoichiometric coefficients of the i reaction (the number of molecules of the $\mathrm{j}$ metabolite, which is consumed $(-)$ or is generated $(+)$ in the i reaction; $v_{i}$ - rate of the i reaction; $F_{j}$ - total flux of $\mathrm{j}$ metabolite (rate of change of metabolite concentration in a cell). In this case, the parameters of the model are the stoichiometric coefficients $\left(S_{i j}\right)$ of the metabolic reactions that are known.

Calculation of elementary flux modes (EFM) from the stoichiometry matrix. An important tool used in FBA is the identification of elementary flux modes (EFMs). EFM is a directed path that includes a minimal and unique set of reactions from one external metabolite to another (Cakir et al., 2004; Schuster \& Kenanov, 2005). EFM analysis allows one to detect and analyze important routes in metabolic networks. An important advantage of this technique is that not only the optimal route but also the full set of possible routes is taken into consideration, thereby accounting for redundancy and flexibility in the metabolic network. EFM was calculated and analyzed in CellNetAnalyser 2017 and Metatool 4.3.

Calculation of control-effective flux coefficients (CEF). The importance of each reaction for the effective and flexible operation of the entire metabolic system was determined using control-effective fluxes (CEFs). CEFs are directly determined from the EFM set by the algorithm given in (Stelling et al., 2002; Cakir et al., 2004; Schwartz et al., 2015). The calculation of CEFs consists in estimating the efficiency of elementary modes (EMs), towards a given cellular objective. The contributions of all EMs are then weighed accordingly and summed up to predict the flux distribution, under the assumption that more efficient routes are likely to be preferential. Cakir et al. (2004) investigated the CEF metabolic network of erythrocytes under conditions of enzymatic insufficiency in the presence of one substrate (glucose). In this paper, the technique of calculating CEF to a more complex metabolic network, where the inputs of several substrates (glucose, SAM, adenosine and amino acids used for the synthesis of glutathione), is applied.

The most ambiguous moment in the CEF theory is the choice of reactions that will be a part of the cellular objective. In pioneering studies (Stelling et al., 2002), which considered simple metabolic networks, these were reactions that produced biomass, or ATP. When applying the CEF approach to complex metabolic networks, the choice of these reactions (the reactions required for cell functioning - cellular objective) affects the CEF value, since the efficiency of each EM is estimated by determining its contribution towards the objective relative to the costs required to establish the mode. This is a certain disadvantage of the CEF approach. Schwartz et al. (2015) suggest comparing CEF calculations using various objective functions with experimental or literary data. In this paper, the reactions that produce or consume metabolites are selected as part of an cellular objective that directly affect the time life of erythrocytes, namely, the reactions associated with the synthesis and transformation of glutathione ( $\mathrm{r} 43-\mathrm{r} 46)$, the methemoglobin reduction (r49e), phosphorylation and ATPase processes (Table 1).

Efficiency value was assigned to each elementary mode for each cellular objective (cj, CELLOBJ) using the following equation:

$$
\varepsilon_{j, \text { CELLOB, }}=\frac{r_{C E L L O B J}^{j}}{\sum_{i}\left|r_{i}^{j}\right|}
$$

$r_{C E L L O B J}^{j}-$ is the yield of production of the cellular objective by EFM (reactions related to cellular objectives), $\sum_{i}\left|r_{i}^{j}\right|^{-}$is the sum of the participation coefficients of each reaction in a certain EFM (the sum of absolute fluxes of each mode), $\mathrm{j}$ - is the EFM index, $\mathrm{i}$ - is the index of a flux through the specific reaction in certain EFM. To calculate $\varepsilon_{j, \text { CELLOBJ }}, r_{i}^{j}$ of the fluxes reactions that are considered to be necessary for the viability of maintenance of erythrocytes were used (GSSGR, GSHpox, GSHox, MemPhos, ATPase, MetHbRed i 23DPGband, GSHSase).

The $C E F\left(v_{i}\right)$ of each reaction $r_{i}^{j}$ was defined as the mean flux through this reaction in all EFMs, where the flux through each mode is weighted with its efficiency (Cakir et al., 2004; Sajitz-Hermstein \& Nikoloski, 2013).

$$
C E F\left(v_{i}\right)=\sum_{C E L L O B J} \frac{1}{r_{C E L L O B J}^{\max }} \cdot \frac{\sum_{j} \varepsilon_{j, C E L L O B J}\left|r_{i}^{j}\right|}{\sum_{j} \varepsilon_{j, C E L L O B J}},
$$


where $r_{C E L L O B J}^{\max }$ is the maximum participation rate in the EFM for reactions that are the part of the cellular objective, $\sum_{j} \varepsilon_{j, \text { CELLOBJ }}\left|r_{i}^{j}\right|$ is the sum of the products of the participation coefficients of each reaction for all EFMs and the corresponding efficiency values, $\sum \varepsilon_{j, \text { CELLOBJ }}$ is the total value of the efficiency of the reactions of cellular objective for each mode.

To determine the behaviour of the entire metabolic system under the condition of a particular enzyme's deficiency, the coefficients of the reaction participation $r_{i}^{j}$ in the EFM that include the deficient enzyme were multiplied by the constant $d_{j}$ to reflect the level of enzyme inactivation, with values from 1 to 0 , where 1 is complete inactivation of the enzyme.

To analyze the effects of enzymopathy on the erythrocyte metabolism, the relative values of CEFs, representing the ratio of reactions CEFs in the presence and absence of the enzyme inactivation were used. Efficiency profiles of the enzymatic reactions upon the degree of GPI deficiency are obtained using CEF approach which predict the redistribution of metabolic fluxes in the loss of enzyme activity. The obtained dependencies were analyzed by comparison with the literature sources.

\section{Results}

For the developed model of metabolism, that consists of 50 reactions (Table 1), 486 elementary fluxes have been calculated. 61 of them use only glucose as a substrate, 115 only SAM and 113 - as an energy source use adenosine. Notably, all fluxes end in a formation of pyruvate and lactate and produce ATP. In the case of using SAM or ADO as a product, hypoxanthine is produced and then extracted from the cell. Adenosine degrades to hypoxanthine (removed from the cells) and ribose-1-phosphate, which is converted in the pentose phosphate pathway into glycolytic intermediates. The fluxes starting with glucose, transform it completely into the ATP, which means that glucose is the main source of energy. 70 elementary modules use as a source of energy glucose and adenosine in different proportions. 83 use glucose and SAM. In 105 elementary pathways, glutathione is synthesized and metabolized. In the case of PGI complete inactivation, 298 elementary fluxes were calculated, indicating the redundancy of the system. In 68 of them, glutathione is synthesized.

The simulation results show once again that the analysis of elementary pathways allows one to draw conclusions about biochemical systems with a limited amount of source information. The pathways that use adenosine or SAM convert part of this metabolite to hypoxanthine, resulting in a fraction of energy dissipates. Thus, the latter cannot be considered the pathways for ATP recovery. These pathways also serve for the transport of purine by erythrocytes (Schuster \& Kenanov, 2005).

Figure 2 shows CEF values for metabolic network reactions in nondeficient case. Reaction numbers on Figure 2 and Table 1 coincide. Reactions from various metabolic cycles are marked by colours. Notably, the CEF value also shows the magnitude of the flux through the reaction, or the extent of the reaction participation in the metabolic fluxes. In the glycolytic cycle, this is a GAPDH-controlled reaction (CEF 5.15). It is known that this enzyme not only determines the direction of flux through the glycolytic path, but also involves fluxes of the phosphate pathway and the cycle of purine catabolism in glycolysis. GSSGR has the highest flux rate (CEF - 5.93), which confirms the importance of GSH and NADPH in human erythrocytes' metabolism. The lowest CEFs were obtained for nucleotide metabolism reactions, indicating their salvage role under ATP depletion conditions (Schuster, \& Kenanov, 2005). In general, the CEF values obtained in the absence of enzymopathy do not contradict existing knowledge and can be used to analyze the work of the metabolic network. Figures 3-5 show changes in the control-effective fluxes coefficients (CEFs) for the reactions included in the model, depending on the degree of PGI inactivation. CEFs are normalized with respect to the index for a fully functioning enzyme. On the abscissa: 0 is a fully functional enzyme, 1 is the complete inactivation of the enzyme.

According to the simulation (Fig. 3), the CEF values for the PGI itself are reduced practically linearly from the degree of inactivation of the enzyme. Inactivation of PGI leads to a decrease in flux through the glycolytic pathway. Loss of activity up to $55 \%$ is not critical, as the CEF values for glycolytic enzymes do not exceed 5\%. Subsequent inactivation of the enzyme leads to a sharper decrease in the fluxes through the glycolytic pathway. Complete inactivation of PGI results in a 20\% drop in the glycolytic flux. The smallest effect of PGI inactivation is on the enzymes of the 2,3-diphosphoglycerate shunt, the triosephosphate isomerase (TPI).

Figure 4 shows changes in the flux control coefficients (CEFs) for purine metabolism reactions, depending on the degree of PGI inactivation. The enzymes of adenylate metabolism reactions are very sensitive to this type of enzymopathy and respond differently to the decrease in the PGI activity. Fluxes through MT, SSAH1, IMPase, ADA, PNPase, PRM, AK responses increase up to $25 \%$, before the RGI activity loss does not exceed $55 \%$. These processes lead to the formation of adenosine. Further inactivation of PGI leads to a flow decrease through these pathways. Fluxes through SSAH2, AMPase, PRPPsyn, ADPRT, HGPRT, AdylK are reduced linearly depending on the activity of the PGI. Complete inactivation of PGI results in a 30\% drop in CEF for these enzymes.

Figure 5 shows the change in CEF values for pentose phosphate, glutathione synthesis and other processes important for cellular functions. According to the modeling data, inactivation of PGI results in lowering fluxes through the reactions of the upper part of the PPP, and the reactions of glutathione oxidation-reduction (with a full inactivation of PGI, CEF reduces by $60 \%$ ). Reduction of CEF values for the lower part of PPP reactions is less: $55 \%$ inactivation of PGI leads to a decrease in flux through these reactions to $20 \%$, complete inactivation to $40 \%$. Fluxes through the pathways leading to the synthesis of glutathione in the cell are also inhibited (up to 30\%). The least susceptible to through PGI inactivation were reactions of hemoglobin restoration, phosphorylation, and fluxes through ATPase, associated with the work of this enzyme ion metabolism and RPI.

\section{Discussion}

The adaptation processes resulting in a preferable metabolic state for cells are crucial to guarantee functioning and, consequently, cell survival. The molecular mechanisms of adaptation involve concerted interactions that ultimately induce a modification of the metabolic state, which is characterized by reaction fluxes and metabolite concentrations. Therefore, the ability of the metabolism to adapt to changing conditions, via regulation of its state, can be regarded as a potential for controlling this complex dynamic system. Biochemical reactions do not act alone and, hence, the process of metabolic adaptation is the result of complex interactions among the system components (metabolites and enzymes). Therefore, assessing the extent to which reaction fluxes could be manipulated to control the metabolic state is a nontrivial task, as metabolic control is a systemic phenomenon. Computational modeling can play an important role in the study and understanding of these processes.

Mathematical approaches such as flux balance analysis (FBA), are widely used to predict the phenotypes of different organisms - from bacteria to plants and humans (Sajitz-Hermstein \& Nikoloski, 2013; Schwartz et al., 2015; Klipp et al., 2016). Although FBA is applied successfully to unicellular organisms as objects of biotechnology and metabolic engineering, its use for the study of metabolic fluxes in human cells is more challenging, due to the difficulty of accurate description of exchange paths between cells and their environment.

In this work, the tools of structural metabolic control (calculations of elementary flux modes (EFMs), control-effective fluxes (CEF)) are used to study the metabolic processes in erythrocytes restructuring in enzyme deficient case. The result of the simulation is efficiency profiles of the enzymatic reactions upon the degree of enzyme deficiency, which allows one to determine the contribution of individual reactions to the function performed by the metabolic network. Glucose phosphate isomerase (GPI, EC 5.3.1.9) catalyzes the isomerization reaction of a pyranose six-membered ring in glucose-6-phosphate to a furanose five-membered ring in fructose-6-phosphate. An important feature of identifying a GPI defective enzyme is the change in its physico-chemical properties (thermostability, isoelectric point, and electrophoretic motion) and its kinetic properties (Repiso et al., 2006; Warang et al., 2012; Fermo et al., 2019). 


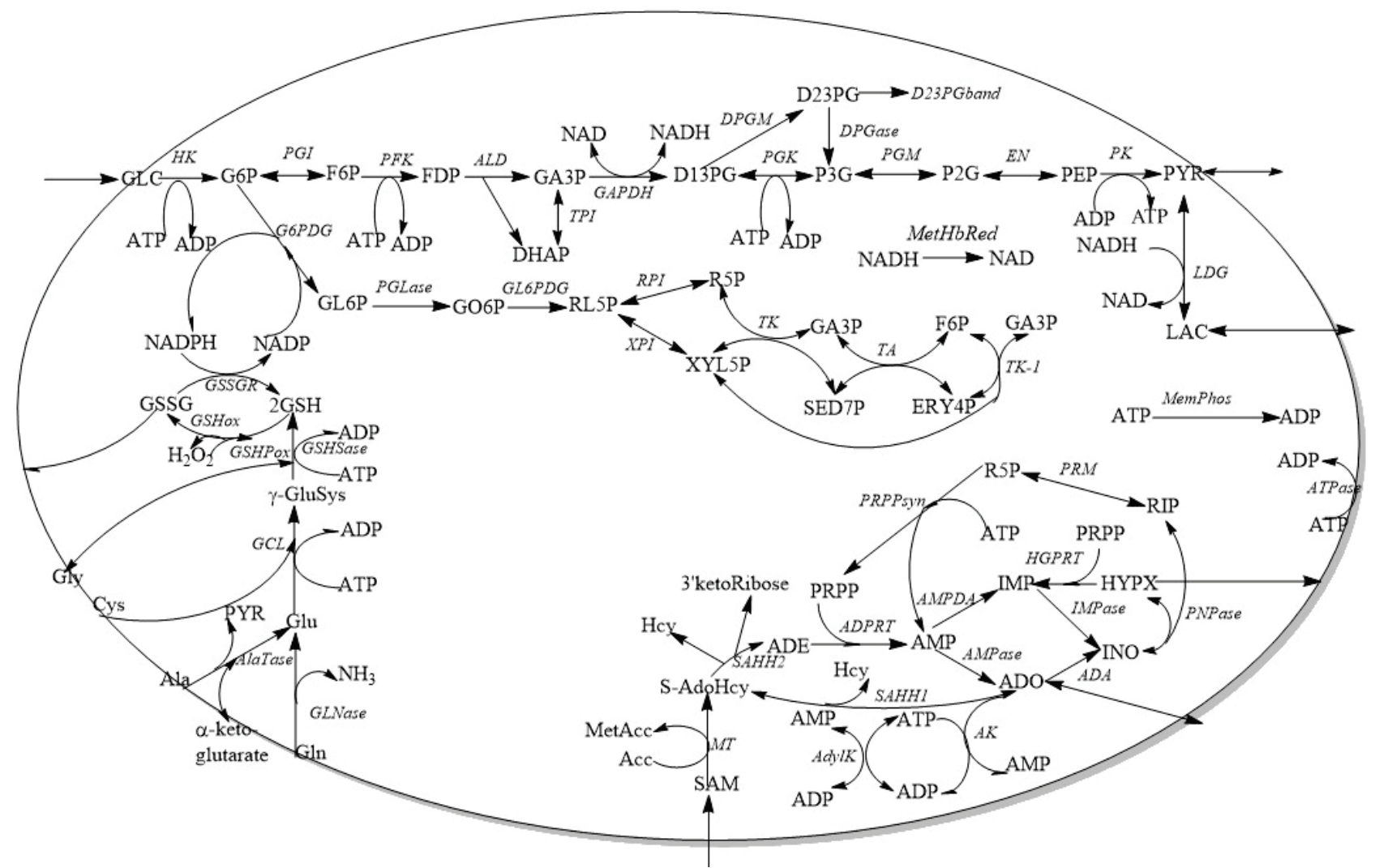

Fig. 1. The human red blood cell metabolism. Adapted from Schuster \& Kenanov, 2005 and Raftos et al., 2010:

Abbreviations for metabolites: ADE, adenine, ADO, adenosine, SAdoHcy, S-adenosylhomocysteine, D13PG, 1,3-biphosphoglycerate, D23PG, 2,3diphosphoglycerate, DHAP, dihydroxyacetone phosphate, ERY4P, erythrose 4-phosphate, F6P, fructose-6-phosphate, FDP, fructose diphosphate, G6P, glucose-6-phosphate, GA3P, glyceraldehydes-3-phosphate, GL6P, 6-phosphogluco lactone, GLC, glucose, GO6P 6-phosphogluconate, GSH, reduced glutathione, GSSG, oxidized glutathione, Hcy, homocysteine, H2O2, hydrogen peroxide, HYPX, hypoxanthine, IMP, inosine monophosphate, INO, inosine, LAC, lactate, P2G, 2-phosphoglycerate, P3G, 3-phosphoglycerate, PEP, phosphenolpyruvate, PRPP, 5-phosphoribosyl pyrophosphate, PYR, pyruvate, R1P, ribose-1-phosphate, R5P, ribose-5-phosphate, RL5P, ribulose-5-phosphate, SAM, S-adenosylmethionine, SED7P, sedoheptulose 7phosphate, XYL5P, xylose-5-phosphate, Gly, glycine, Gln, glutamine, Glu, glutamic acid, Cys, cysteine. Abbreviations for reactions: AdylK, adenylate kinase (EC 2.7.4.3), ADA, adenosine deaminase (EC 3.5.4.4), ADPRT, adenine phosphoribosyltransferase (EC 2.4.2.7), AK, adenosine kinase (EC 2.7.1.20), AlaTase, alanine aminotransferase (EC 2.6.1.2); ALD, aldolase (EC 4.1.2.13), AMPase, adenosine monophosphate phosphohydrolase (EC 3.1.3.5), AMPDA, adenosine monophosphate deaminase (EC 3.5.4.6), DPGase, diphosphoglycerate phosphatase (EC 3.1.3.13), DPGM, diphosphoglycerate mutase (EC 5.4.2.4), EN, enolase (EC 4.2.1.11), GCL, $\gamma$-glutamate-cysteine ligase (EC 6.3.2.2), G6PDH, glucose-6-phosphate dehydrogenase (EC 1.1. 1.49), GAPDH, glyceralde-hyde-3-phosphate dehydrogenase (EC 1.2.1.12), GLNase, glutaminase (EC 3.5.1.2), GL6PDH, phosphogluconate dehydrogenase (EC 1.1.1.44), GSHSase, glutathione synthetase (EC 6.3.2.3), GSSGR, glutathione-disulfide reductase (EC 1.8.1.7), HGPRT, hypoxanthine phosphoribosyltransferase (EC 2.4.2.8), HK, hexokinase (EC 2.7.1.1), IMPase, inosine monophosphate phosphohydrolase (EC 3.1.3.5), LDH, lactate dehydrogenase (EC 1.1.1.27), MemPhos, membrane phosphorylation, MetHbRed, methemoglobin reductase, MT, Methyl-transferases, PFK, phosphofructokinase (EC 2.7.1.11), PGI, glucose-6-phosphate isomerase (EC 5.3.1.9), PGK, phosphoglycerate kinase (EC 2.7.2.3), PGLase, phosphogluconolactonase (EC 3.1.1.31), PGM, phosphoglycerate mutase (EC 5.4.2.1), PK, pyruvate kinase (EC 2.7.1.40), PNPase, purine-nucleoside phosphorylase (EC 2.4.2.1), PRM, phosphoribomutase (EC 5.4.2.7), PRPPsyn, phosphoribosylpyro-phosphate synthetase (EC 2.7.6.1), RPI, ribose-5-phosphate isomerase (EC 5.3.1.6), SAHH1, SAHH2, S-Adenosylhomocysteine hydrolase (EC 3.3.1.1), TA, transaldolase (EC 2.2.1.2), TK, transketolase (EC 2.2.1.1), TPI, triosephosphate isomerase (EC 5.3.1.1), XPI, ribulose phosphate epimerase (EC 5.1.3.1)

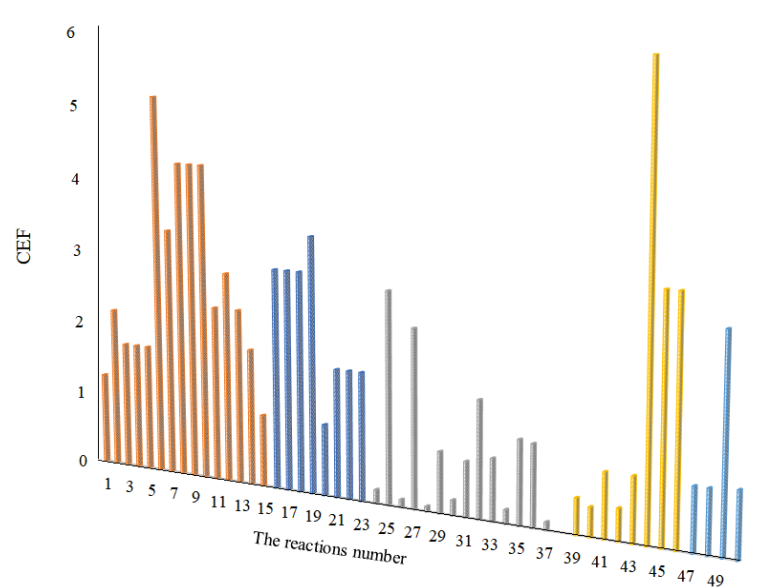

Fig. 2. The absolute values $\mathrm{CEF}$ for metabolic network reactions in non-deficient case

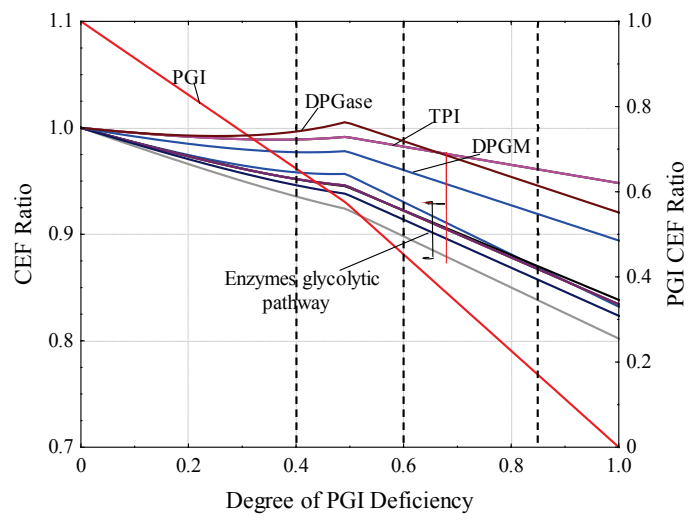

Fig. 3. Changes of glycolysis control-effective fluxes upon the change of PGI activity: Y-axis indicates CEF ratios with respect to the normal case; in $\mathrm{x}$-axis, 0 shows fully functional enzyme, 1 means a complete deficiency of the enzyme 


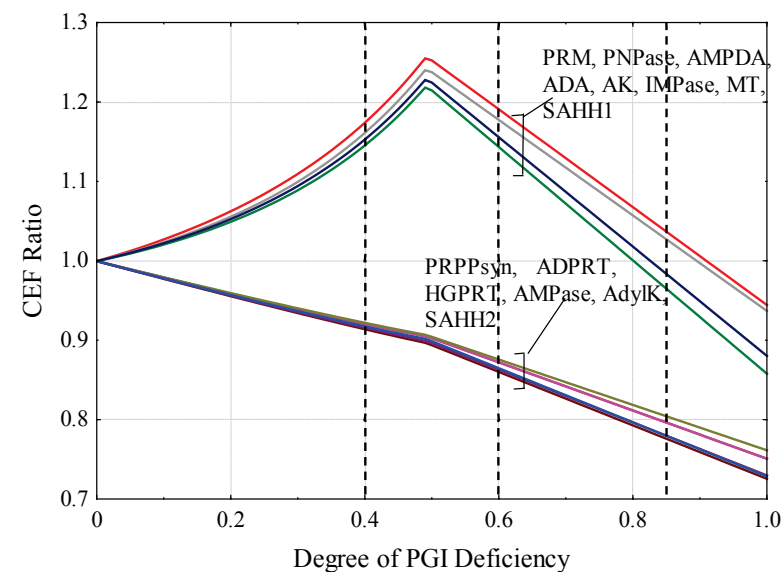

Fig. 4. Changes in adenylate metabolism cycle control-effective fluxes upon the change of PGI activity

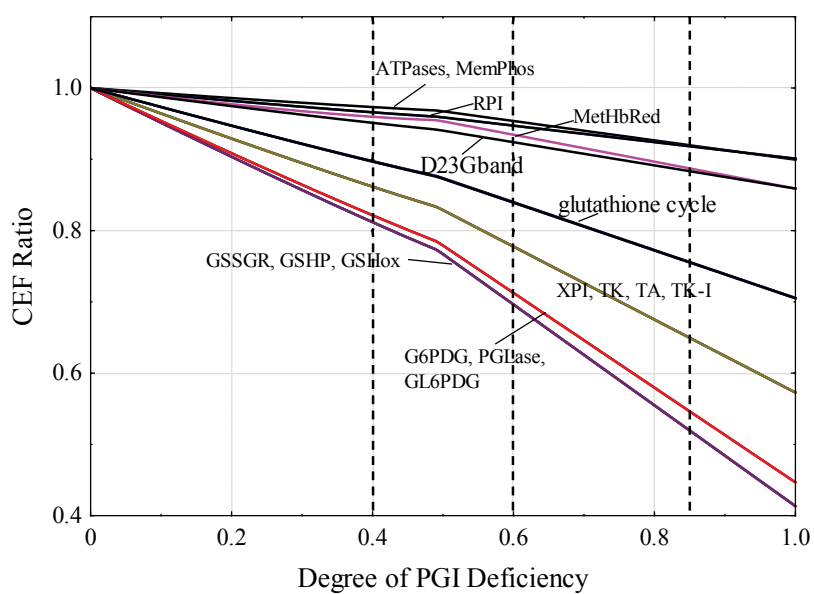

Fig. 5. Changes in pentose phosphate pathway, glutathione synthesis pathway and cellular objective reactions control-effective fluxes upon the change of PGI activity

When analyzing the severity of the disease in the case of an unstable mutant enzyme, it is important to know not only the average value of its activity in the population of erythrocytes, but also the time of its inactivation, which determines the erythrocyte's life span. Energy depletion and metabolic impairment lead to a shortening of the mature erythrocytes' functioning time. The ability to compensate for deficiency of the enzyme due to excessive isozymic expression or the use of alternative pathways contributes to the positive clinical picture of patients with enzymopathies. Understanding the redistribution of metabolic flows in the erythrocytes can provide a basis for developing new therapeutic strategies aimed to increase the cells' life span.

In addition, a considerable amount of new data on the peculiarities of the activity of the erythrocyte metabolic networks has appeared at the present time, and an understanding of the complexity of the organization and the importance of their functions (other than the oxygen transport function) for the whole organism has been formed. Creating a model contributes to the systematization of a large number of experimental data and allows hypotheses to be tested about the peculiarities of the regulation of metabolic processes in these cells, as well as formulation of new hypotheses.

It is believed that the conversion of glucose through the glycolytic pathway is the only source of NADH and ATP in the erythrocytes. $\mathrm{NADH}$ is a major reducing agent for hemoglobin. When NADH is used for methemoglobin reduction, the total flux via glycolysis is enhanced, with pyruvate or lactate as the final product. In the case of GPI deficiency, the G6P/F6P ratio in erythrocytes is elevated (Kugler \& Lakomek 2000; Warang et al., 2012; Mojzikova et al., 2018), and this fact is not a consequence of reticulocytosis (Merkle \& Pretsch, 1993). Increased level of glucose-6-phosphate causes reverse inhibition of hexokinase (HK), an enzyme that catalyzes the conversion of glucose to glucose-6-phosphate (van Wijk \& van Solinge, 2005; Rossi et al., 2012). In the case of very low HK activity, both the generation of NADH as well as the supply of G6P to the PPP, and thereby the generation of NADPH, will be seriously hampered (van Zwieten et al., 2013). The result of these processes should be depletion of cells with ATP (Kugler \& Lakomek, 2000; Warang et al., 2012), although the decrease in ATP content may not occur (Merkle \& Pretsch, 1993).

\section{Table 1}

Reactions in erythrocyte's metabolism and corresponding catalyzing enzymes (Arrow type, $\rightarrow$ or $\leftrightarrow$, indicates whether the reaction is considered irreversible or reversible in the analysis, $\mathrm{N}$-reactions' numbers in the model)

\begin{tabular}{|c|c|c|}
\hline $\mathrm{N}$ & Reactions & Enzymes \\
\hline \multicolumn{3}{|c|}{ Glycolysis } \\
\hline 1 & $1 \mathrm{Glc}+1 \mathrm{ATP} \rightarrow 1 \mathrm{G} 6 \mathrm{P}+1 \mathrm{ADP}$ & HK \\
\hline 2 & 1 G6P 1 F6P & PGI \\
\hline 3 & $1 \mathrm{~F} 6 \mathrm{P}+1 \mathrm{ATP} \rightarrow 1 \mathrm{FDP}+1 \mathrm{ADP}$ & PFK \\
\hline 4 & $1 \mathrm{FDP} \leftrightarrow 1 \mathrm{GA} 3 \mathrm{P}+1 \mathrm{DHAP}$ & ALD \\
\hline 5 & 1 DHAP $\leftrightarrow 1$ GA3P & TPI \\
\hline 6 & $1 \mathrm{GA} 3 \mathrm{P}+1 \mathrm{NAD}{ }^{+} \leftrightarrow 1 \mathrm{D} 13 \mathrm{PG}+1 \mathrm{NADH}$ & GAPDH \\
\hline 7 & $1 \mathrm{D} 13 \mathrm{PG}+1 \mathrm{ADP} \leftrightarrow 1 \mathrm{P} 3 \mathrm{G}+1 \mathrm{ATP}$ & PGK \\
\hline 8 & $1 \mathrm{P} 3 \mathrm{G} \leftrightarrow 1 \mathrm{P} 2 \mathrm{G}$ & PGM \\
\hline 9 & $1 \mathrm{P} 2 \mathrm{G} \leftrightarrow 1 \mathrm{PEP}$ & EN \\
\hline 10 & $1 \mathrm{PEP}+1 \mathrm{ADP} \rightarrow 1 \mathrm{PYR}+1 \mathrm{ATP}$ & PK \\
\hline 11 & $1 \mathrm{PYR}+1 \mathrm{NADH} \rightarrow 1 \mathrm{LAC}+1 \mathrm{NAD}^{+}$ & LDG \\
\hline 12 & 1 PYR $=1$ PYRext & - \\
\hline 13 & $1 \mathrm{LAC}=1 \mathrm{LACext}$ & - \\
\hline \multicolumn{3}{|c|}{ 2,3-bisphosphoglycerate shunt } \\
\hline 14 & $1 \mathrm{D} 13 \mathrm{PG} \rightarrow 1 \mathrm{D} 23 \mathrm{PG}$ & DPGM \\
\hline 15 & $1 \mathrm{D} 23 \mathrm{PG} \rightarrow 1 \mathrm{P} 3 \mathrm{G}$ & DPGase \\
\hline \multicolumn{3}{|c|}{ Pentose phosphate pathway } \\
\hline 16 & $1 \mathrm{G} 6 \mathrm{P}+1 \mathrm{NADP} \rightarrow 1 \mathrm{GL} 6 \mathrm{P}+1 \mathrm{NADPH}$ & G6PDH \\
\hline 17 & 1 GL6P $\leftrightarrow 1$ GO6P & PGLase \\
\hline 18 & $1 \mathrm{GO} 6 \mathrm{P}+1 \mathrm{NADP} \rightarrow \mathrm{RL} 5 \mathrm{P}+\mathrm{NADPH}$ & GL6PDH \\
\hline 19 & $1 \mathrm{RL} 5 \mathrm{P} \leftrightarrow 1 \mathrm{XYL} 5 \mathrm{P}$ & XPI \\
\hline 20 & 1 RL5P $\leftrightarrow 1$ R5P & RPI \\
\hline 21 & $1 \mathrm{XYL} 5 \mathrm{P}+1 \mathrm{R} 5 \mathrm{P} \leftrightarrow 1 \mathrm{SED} 7 \mathrm{P}+1 \mathrm{GA3P}$ & TK 1 \\
\hline 22 & 1SED7P +1 GA3P $\leftrightarrow 1$ F6P +1 ERY4P & TA \\
\hline 23 & $1 \mathrm{XYL} 5 \mathrm{P}+1 \mathrm{ERY} 4 \mathrm{P} \leftrightarrow 1 \mathrm{~F} 6 \mathrm{P}+1 \mathrm{GA} 3 \mathrm{P}$ & TK \\
\hline \multicolumn{3}{|c|}{ Nucleotide metabolism } \\
\hline 24 & $1 \mathrm{R} 5 \mathrm{P}+1 \mathrm{ATP} \rightarrow 1 \mathrm{PRPP}+1 \mathrm{AMP}$ & PRPPsyn \\
\hline 25 & $1 \mathrm{R} 1 \mathrm{P} \leftrightarrow 1 \mathrm{R} 5 \mathrm{P}$ & PRM \\
\hline 26 & $1 \mathrm{PRPP}+1 \mathrm{ADE} \rightarrow 1 \mathrm{AMP}$ & ADPRT \\
\hline 27 & $1 \mathrm{INO} \leftrightarrow 1 \mathrm{HYPX}+1 \mathrm{R} 1 \mathrm{P}$ & PNPase \\
\hline 28 & $1 \mathrm{HYPX}+1 \mathrm{PRPP} \rightarrow 1 \mathrm{IMP}$ & HGPRT \\
\hline 29 & $1 \mathrm{IMP} \rightarrow 1 \mathrm{INO}$ & IMPase \\
\hline 30 & $1 \mathrm{AMP} \rightarrow 1 \mathrm{ADO}$ & AMPase \\
\hline 31 & $1 \mathrm{AMP} \rightarrow 1 \mathrm{IMP}$ & AMPDA \\
\hline 32 & $1 \mathrm{ADO} \rightarrow 1 \mathrm{INO}$ & ADA \\
\hline 33 & $1 \mathrm{ADO}+1 \mathrm{ATP} \rightarrow 1 \mathrm{ADP}+1 \mathrm{AMP}$ & AK \\
\hline 34 & $2 \mathrm{ADP} \leftrightarrow 1 \mathrm{ATP}+1 \mathrm{AMP}$ & AdylK \\
\hline 35 & $\mathrm{SAM}+\mathrm{Acc} \rightarrow \mathrm{S}-\mathrm{AdoHcy}+\mathrm{MetAcc}$ & MT \\
\hline 36 & S-AdoHcy $\rightarrow$ ADO+Hcy & SAHH1 \\
\hline 37 & S-AdoHcy $\rightarrow$ ADE + Hcy +3 'ketoRibose & SAHH2 \\
\hline 38 & ADO_ext $\rightarrow$ ADO & - \\
\hline \multicolumn{3}{|c|}{ Glutathione synthesis } \\
\hline 39 & Ala $+\alpha$-ketoglutarate $\rightarrow$ PYR + Glu & AlaTase \\
\hline 40 & Gln $\rightarrow$ Glu & GLNase \\
\hline 41 & $\mathrm{ATP}+\mathrm{Cys}+\mathrm{Glu} \rightarrow \mathrm{ADP}+\mathrm{GluCys}$ & GCL \\
\hline 42 & GSSG $\rightarrow$ GSSG_ext & - \\
\hline \multicolumn{3}{|c|}{ Cellular functions } \\
\hline 43 & $\mathrm{ATP}+\mathrm{Gly}+\mathrm{GluCys} \rightarrow \mathrm{ADP}+\mathrm{GSH}$ & GSHSase \\
\hline 44 & $1 \mathrm{GSSG}+1 \mathrm{NADPH} \leftrightarrow 2 \mathrm{GSH}+1 \mathrm{NADP}$ & GSSG-R \\
\hline 45 & $2 \mathrm{GSH}+1 \mathrm{H}_{2} \mathrm{O}_{2} \rightarrow 1 \mathrm{GSSG}$ & GSHpox \\
\hline 46 & $2 \mathrm{GSH}+1 \mathrm{O}_{2} \rightarrow 1 \mathrm{GSSG}+1 \mathrm{H}_{2} \mathrm{O}_{2}$ & GSHox \\
\hline 47 & $1 \mathrm{ATP} \rightarrow 1 \mathrm{ADP}$ & ATPase \\
\hline 48 & $1 \mathrm{ATP} \rightarrow 1 \mathrm{ADP}$ & MemPhos \\
\hline 49 & $1 \mathrm{NADH} \rightarrow 1 \mathrm{NAD}$ & MetHbRed \\
\hline 50 & $\mathrm{D} 23 \mathrm{PG}+\mathrm{Hb} \rightarrow \mathrm{D} 23 \mathrm{PG}-\mathrm{Hb}$ & D23PGband \\
\hline
\end{tabular}


In this regard, the model provided additional mechanisms based on the use of S-adenosylmethionine (SAM) and adenosine as alternative energy sources. Beyond this, SAM is an important methyl donor in many transmethylation reactions (Altintas \& Sezgin, 2004), whose biological function is associated with the regeneration or degradation of agedamaged proteins. Methyl ester formation in erythrocyte membrane proteins has also been used as a marker reaction to tag these abnormal residues and to monitor their increase associated with erythrocyte ageing diseases. Human erythrocytes contain several protein methyl transferases that catalyze the esterification of side chain carbonyl groups (Reisz et al., 2018). The paper shows 115 modes that could use SAM as an energy source. This may to some extent explain the excessive intake of folic acid in patients with anemia (Adama van Scheltema et al., 2015). Based on the analysis of EFMs, it can be argued that the infrequently mentioned route through SAHH is very important and provides additional opportunities for ATP formation (Schuster \& Kenanov, 2005). Another possibility for ATP replenishment is to use adenosine. It is known that extracellular enzymes such as ecto-5'-nucleotidase (CD73) and ATPase/CD39 are part of the regulatory control of adenine nucleotide in erythrocytes. The extracellular AMP may also be dephosphorylated to adenosine by CD73 (Sun, et al., 2017; Adebiyi et al., 2019). Adenosine is transmitted through nucleoside transporters, such as ENT1/2, and rapidly catabolized by intracellular adenosine deaminase (ADA).

By adding to the model additional ATP salvage pathways, we obtained results that coincide with the experimental data, namely, a decrease in the glycolytic pathway by $20 \%$ under full GPI inactivation. The ribose-1-phosphate formed from inosine in the catabolism of adenosine to hypoxanthine (Fig. 1) can be incorporated into glycolysis through reactions of the pentose phosphate pathway (Clarke et al., 2003; Paglia et al., 2016) and thereby increase the rate of glycolytic production of ATP from ADP in cells. The relationship between the rate of increase of the adenylate pool and the adenosine entry has been investigated (Komarova et al., 1999). This can be explained by the fact that the CEF values for lower PPP reactions are lower than for the reactions of the upper PPP (Fig. 5).

The results of the study indicate that salvage pathways using SAM and adenosine have relatively high levels of redundancy. This fact can be considered as a theoretical explanation of clinical observations that many enzyme deficiencies, including PGI, are usually not critical. The simulation results indicate that adenosine formation and catabolism pathways are important in the erythrocytes' energy metabolism. Fluxes through MT, SSAH1, IMPase, ADA, PNPase, PRM, AK increase up to $25 \%$ to the $55 \%$ margin of PGI inactivation (Fig. 4). Adenosinebased signaling can regulate the ability of erythrocytes to deliver $\mathrm{O}_{2}$ by increasing production of $\mathrm{D} 23 \mathrm{PG}$, which provides a new way of maintaining levels of D23PG. Intracellular signaling pathways, including purinergic and sphingolipid signaling networks, promote metabolism reprogramming, directing glucose metabolism to glycolysis through a pentose phosphate partway. These processes are investigated with hypoxia (Sun, et al., 2017). The activation of ADORA2B receptors led to the sequential activation of AMP-activated protein kinase (AMPK; a well-known cellular energy sensor) and diphosphoglycerate mutase (DPGM), resulting in increased D23PG production. The model includes 113 elemental modes that can only be initiated by adenosine. The results of the calculations show that the D23PG shunt reactions maintain high CEF values under GPI inactivation conditions (Fig. 3).

Notably, pathways using S-adenosylmethionine or adenine produce hypoxanthine. The reaction of hypoxanthine transformation to IMP has a very low CEF (0.07), which indicates that hypoxanthine is excreted out of the cells and is not an intracellular process. An intense flux of hypoxanthine may be dangerous, since its levels above the threshold value of $100 \mu \mathrm{M}$ may be sufficient to initiate oxidative stress (Casali et al., 2016). In addition, if ATP pool renewal was stimulated by folates, homocysteine is formed as a byproduct and can become a source of toxic products. However, it should be noted that the role of homocysteine in erythrocytes is not unambiguous, it is believed that in erythrocytes, it can partially be metabolized to cysteine, thereby supporting the glutathione synthesis. The model predicts a decreased flux through PPP reactions, and to a greater extent (up to $60 \%$ ) through reactions that produce
NADPH (Fig. 5). NADPH is the main source of reducing equivalents in erythrocytes, used by most defense systems. When NADPH content becomes limited, erythrocytes are susceptible to damage (van Zwieten et al., 2013). Continuous reduction of NADP+ to NADPH is crucial for maintaining high concentrations in red cells of reduced glutathione (GSH) and peroxiredoxins, which serve as reducing agents in all peroxide- and thiol-reducing actions. Therefore, the NADPH-generating pathway is of utmost importance for the reduction capacity of erythrocytes. NADPH can only be formed by converting glucose-6-phosphate (G6P) into 6-phosphoglyunohalactone, under the action of G6PDH, and the successive transformation reaction of 6-phosphogluconolactone to ribulose-5- phosphate, catalyzed by 6PGD (Fig. 1). Lack of NADPH can be a cause of destabilization of the erythrocyte membrane, lysis, and the onset of chronic hemolytic anemia. Lack of reducing agents such as NADPH and GSH in the presence of GPI deficiency may be the cause of a hemolytic crisis due to exogenous oxidants such as infections or drugs (Rossi et al., 2010).

Glutathione (GSH) is a tripeptide synthesized from L-cysteine, L-glutamic acid and glycine (Fig. 1). Glutathione (GSH) is present at high concentrations $(2-10 \mathrm{mM})$ in erythrocytes and acts by itself or via glutathione peroxidase as a major reducing source to remove low concentrations of hydrogen peroxide and lipid/alkyl peroxides. The synthesis of GSH from amino acids is catalyzed by ATP-dependent glutamate cysteine ligase (GCL) and GSH synthase (Fig. 1), which maintains high levels of GSH in erythrocytes. (Raftos et al., 2010; Whillier et al., 2011). The depletion of ATP and NADPH becomes a limiting factor for the synthesis of glutathione (ATP-dependent process), resulting in a decrease in the glutathione pool, which coincides with the results of simulation (Fig. 4). Since the $\gamma$-glutamic cycle is incomplete in mature erythrocytes, the lack of activity of oxoprolinase results in the accumulation of 5-oxoprolin in erythrocytes and plasma, which is a key marker of metabolic age of erythrocytes (Paglia et al., 2016).

Reasons why therapeutic attempts to influence the work of metabolic pathways with inorganic phosphate, methylene blue, and ascorbate were not effective (Kugler \& Lakomek, 2000) could be seen from the simulation results. Inorganic phosphate is involved in phosphorylation reactions, but it decreases activity of hexokinase, which is already decreased because of the GPI deficiency. The introduction of a large amount of inorganic phosphate results in the violation of glucose uptake by red blood cells and an additional decrease in the ATP content. The metabolism of ascorbate in erythrocytes itself is very complex, in red blood cells ascorbate enters in an oxidized form and then is restored by intracellular reductase. In this regard, exogenous ascorbate may not increase intracellular content due to a decrease in the amount of NADPH and GSH, which are substrates for the recovery dehydroascorbate (Dotsenko et al., 2018).

The simulation allows us to formulate the following hypotheses about therapy that could be useful for prolonging the life span of cells:

- stimulation of HK and G6PDG in order to activate PPP pathway and develop NADPH;

- stimulation of the synthesis of glutathione, by raising the amino acids in the blood plasma such as alanine, glutamate, glycine, cysteine, $\mathrm{N}$-acetylcysteine (NAC). It was shown that an increase in the intracellular concentrations of these amino acids (Whillier et al., 2011) by as little as $10 \%$ and intracellular accumulation of NAC to $100 \mu \mathrm{mol} / \mathrm{L}$, was sufficient for a predicted return of the glutathione synthesis rate to normal measured values, in spite of the reduced ATP concentration and reduced transporter activity;

- stimulation of SAM pool by the folate, methionine, vitamins $\mathrm{B}_{6}$ and $\mathrm{B}_{12}$ consumption (Reisz et al., 2018), which will increase the content of ATP in cells, stimulate the processes of methylation of proteins carbonyl groups, transsulfation of homocysteine to cysteine and affect the redistribution of metabolic fluxes in the cell;

- during anemia, SAHH activity is significantly reduced (Altintas $\&$ Sezgin, 2004). This enzyme plays a key role in the methyl esterification (D'Angelo et al., 2013). Since all SAM-dependent methyltransferases are regulated in vivo by the ratio of [SAM]/[SAH], its reduction is a marker for the deterioration of protein methylation processes, dysregulation of nucleic acid formation cycles, and premature ageing of cells 
The activity of this enzyme has not been investigated for GPI enzymopathy, but the results of simulations indicate the possibility of its decreased activity. The activation of SAHH could reduce the oxidative and energy load on cells and protect them from premature ageing;

- Rossi et al. (2010) reported on nonimmune hemolytic anemia caused by amoxicillin in a child with a GPI deficiency. Decreasing the content of reducing equivalents in case of deficiency of this enzyme can lead to impairment of the system that removes free radicals generated by antibiotics, thereby resulting in perioxidative damage to hemoglobin, red blood cell enzymes, and soluble constituents, thereby leading to hemolytic crisis. Therefore, the use of antioxidants, such as vitamin E, is necessary in order to reduce the oxidative load during the administration of antibiotics.

The simulation results show a high concurrence with the literature data. The obtained CEF profiles of reactions help to understand the metabolic state of cells with partial loss of GPI activity. It is known that in heterozygotes, the activity of GPI in erythrocytes is $40-60 \%$ from normal and the disease proceeds asymptomatically (Kugler \& Lakomek, 2000; Warang et al., 2012; Mojzikova et al., 2018), which is well explained by the results of modeling. In this case, the molecular adaptive mechanisms discussed above may contribute to maintaining the content of ATP, although the content of NADPH and GSH will be already reduced. Homozygotes have the activity of this enzyme at only $14-30 \%$ of normal. In that case, a significant decrease in NADPH and GSH is predicted, which is likely to cause premature ageing and hemolytic brittleness. This approach can be applied not only for the analysis of enzymes, but also for the development of drugs aimed at inactivating certain enzymes.

\section{Conclusions}

Analysis of the profiles of enzymatic reactions, obtained using tools of metabolic structural analysis suggests that erythrocytes are capable of metabolizing other substrates (SAM, adenosine), which helps to overcome the effects of energy stress in the case of enzymopathies. GPI enzymopathy has been shown to reduce flow through glycolysis and pentose phosphate pathways, resulting in a decrease in the content of reducing agents such as NADPH and GSH, ATP. The decline in the synthesis of GSH and SAHH activity is predicted. These processes can cause hemolytic crisis in presence of exogenous oxidants such as infections or drugs. Based on the results of the simulation, hypotheses have been developed regarding the therapy strategies which may be useful for prolonging the life of cells.

\section{References}

Adama van Scheltema, P. N., Zhang, A., Ball, L. M., Steggerda, S. J., van Wijk, R., Fransen van de Putte, D. E., \& van Kamp, I. L. (2015). Successful treatment of fetal hemolytic disease due to glucose phosphate isomerase deficiency (GPI) using repeated intrauterine transfusions: A case report. Clinical Case Reports, 3, 862-865.

Adebiyi, M. G., Manalo, J. M., \& Xia, Y., (2019). Metabolomic and molecular insights into sickle cell disease and innovative therapies. Blood Advances, 3(8), 1347-1355.

Altintas, E., \& Sezgin, O. (2004). S-adenosylhomocysteine hydrolase, S-adenosylmethionine, S-adenosylhomocysteine: Correlations with ribavirin induced anemia. Medical Hypotheses, 63(5), 834-837.

Burger, N. C. M., van Wijk, R., Bresters, D., \& Schell, E. A. (2019). A novel mutation of glucose phosphate isomerase (GPI) causing severe neonatal anemia due to GPI deficiency. Journal of Pediatric Hematology/Oncology, 41(3), 186-189.

Cakir, T., Tacer, C. S., \& Ulgen, K. O. (2004). Metabolic pathway analysis of enzyme-deficient human red blood cells. Biosystems, 78, 49-67.

Casali, E., Berni, P., Spisni, A., Baricchi, R., \& Pertinhez, T. A. (2016). Hypoxanthine: A new paradigm to interpret the origin of transfusion toxicity. Blood Transfusion, 14(6), 555-556.

Chaturvedi, S., Singh, A. K., Keshari, A. K., Maity, S., Sarkar, S., \& Saha, S. (2016). Human metabolic enzymes deficiency: A genetic mutation based approach. Scientifica, 2016, e9828672.

Clarke, J. L., Vulliamy, T. J., Roper, D., Mesbah-Namin, S. A., Wild, B. J., Walker, J. I., Will, A. M., Bolton-Maggs, P. H., Mason, P. J., \& Layton D. M. (2003). Combined glucose-6-phosphate dehydrogenase and glucosephosphate isomerase deficiency can alter clinical outcome. Blood Cells, Molecules and Diseases, 30, 258-263.

D'Angelo, S., Trojsi, F., Salvatore, A., Daniele, L., Raimo, M., Galletti, P., \& Monsurrò, M. R. (2013). Accumulation of altered aspartyl residues in erythrocyte membrane proteins from patients with sporadic amyotrophic lateral sclerosis. Neurochemistry International, 63(6), 626-634.

Dotsenko, O. I., Taradina, G. V., \& Voronych, M. V. (2018). Enzyme protection systems of erythrocytes in conditions of ascorbate recirculation and oxidative loading. Regulatory Mechanisms in Biosystems, 9(4), 584-590.

Durmuş Tekir, S., Cakir, T., \& Ulgen, K. O. (2006). Analysis of enzymopathies in the human red blood cells by constraint-based stoichiometric modeling approaches. Computational Biology and Chemistry, 30, 327-338.

Fermo, E., Vercellati, C., Marcello, A. P., Zaninoni, A., Aytac, S., Cetin, M., Capolsini, I., Casale, M., Paci, S., Zanella, A., Barcellini, W., \& Bianchi, P. (2019). Clinical and molecular spectrum of glucose-6-phosphate isomerase deficiency. Report of 12 new cases. Frontiers in Physiology, 2019, 10.

Grace, R. F., \& Glader, B. (2018). Red blood cell enzyme disorders. Pediatric Clinics of North America, 65(3), 579-595.

Jamwal, M., Aggarwal, A., Das, A., Maitra, A., Sharma, P., Krishnan, S., Arora, N., Bansal, D., \& Das, R. (2017). Next-generation sequencing unravels homozygous mutation in glucose-6-phosphate isomerase, GPIc.1040G $>$ A (p.Arg347His) causing hemolysis in an Indian infant. Clinica Chimica Acta, 468, 81-84.

Klipp, E., Liebermeister, W., Wierling, C., \& Kowald, A. (2016). System biology: A textbook. Wiley-Blackwell.

Komarova, S. V., Mosharov, E. V., Vitvitsky, V. M., \& Ataullakhanov, F. I. (1999). Adenine nucleotide synthesis in human erythrocytes depends on the mode of supplementation of cell suspension with adenosine. Blood Cells, Molecules and Diseases, 25(3-4), 170-179.

Kugler, W., \& Lakomek, M. (2000). Glucose-6-phosphate isomerase deficiency. Best Practice and Research Clinical Haematology, 13, 89-101.

Lin, H. Y., Liu, J. H., Cheng, K. L., Lin, J. Y., Liu, N. R., \& Meng, M. (2015). A novel binding of GTP stabilizes the structure and modulates the activities of human phosphoglucose isomerase/autocrine motility factor. Biochemistry and Biophysics Reports, 30(2), 14-22.

Mairbäurl, H. (2018). Neocytolysis: How to get rid of the extra erythrocytes formed by stress erythropoiesis upon descent from high altitude. Frontiers in Physiology, 9, 345-351.

Merkle, S., \& Pretsch, W. (1993). Glucose-6-phosphate isomerase deficiency associated with nonspherocytic hemolytic anemia in the mouse: An animal model for the human disease. Blood, 81(1), 206-213.

Mojzikova, R., Koralkova, P., Holub, D., Saxova, Z., Pospisilova, D., Prochazkova, D., Dzubak, P., Horvathova, M., \& Divoky, V. (2018). Two novel mutations (p.(Ser160Pro) and p.(Arg472Cys)) causing glucose-6-phosphate isomerase deficiency are associated with erythroid dysplasia and inappropriately suppressed hepcidin. Blood Cells, Molecules and Diseases, 69, 23-29.

Paglia, G., D'Alessandro, A., Rolfsson, Ó., Sigurjónsson, Ó. E., Bordbar, A., Palsson, S., Nemkov, T., Hansen, K. C., Gudmundsson, S., \& Palsson, B. O. (2016). Biomarkers defining the metabolic age of red blood cells during cold storage. Blood, 128(13), 43-50.

Raftos, J. E., Whillier, S., \& Kuchel, P. W. (2010). Glutathione synthesis and turnover in the human erythrocyte: Alignment of a model based on detailed enzyme kinetics with experimental data. Journal of Biological Chemistry, 285(31), 23557-23567.

Reisz, J. A., Nemkov, T., Dzieciatkowska, M., Culp-Hill, R., Stefanoni, D., Hill, R. C., Yoshida, T., Dunham, A., Kanias, T., Dumont, L. J., Busch, M., Eisenmesser, E. Z., Zimring, J. C., Hansen, K. C., \& D'Alessandro, A. (2018). Methylation of protein aspartates and deamidated asparagines as a function of blood bank storage and oxidative stress in human red blood cells. Transfusion, 58(12), 2978-2991.

Rossi, F., Ruggiero, S., Gallo, M., Simeone, G., Matarese, S. M., \& Nobili, B. (2010). Amoxicillin-induced hemolytic anemia in a child with glucose-6-phosphate isomerase deficiency. Annals of Pharmacotherapy, 44(7-8), 1327-1329.

Sajitz-Hermstein, M., \& Nikoloski, Z. (2013). Structural control of metabolic flux. PLoS Computational Biology, 9(12), e1003368.

Schuster, S., \& Kenanov, D. (2005). Adenine and adenosine salvage pathways in erythrocytes and the role of S-adenosylhomocysteine hydrolase. A theoretical study using elementary flux modes. FEBS Journal, 272, 5278-5290.

Schwartz, J.-M., Barber, M., \& Soons, Z. (2015). Metabolic flux prediction in cancer cells with altered substrate uptake. Biochemical Society Transactions, 43, 1177-1181.

Stelling, J., Klamt, S., Bettenbrock, K., Schuster, S., \& Gilles, E. D. (2002). Metabolic network structure determines key aspects of functionality and regulation. Nature, 420, 190-193.

Sun, K., Liu, H., Song, A., Manalo, J. M., D'Alessandro, A., Hansen, K. C., Kellems, R. E., Eltzschig, H. K., Blackburn, M. R., Roach, R. C., \& Xia, Y. (2017). Erythrocyte purinergic signaling components underlie hypoxia adaptation. Journal of Applied Physiology, 123, 951-956. 
van Wijk, R., \& van Solinge, W. W. (2005). The energy-less red blood cell is lost: Erythrocyte enzyme abnormalities of glycolysis. Blood, 106(13), 40344042.

van Zwieten, R., Verhoeven, A. J., \& Roos, D. (2013). Inborn defects in the antioxidant systems of human red blood cells. Free Radical Biology and Medicine, 67, 377-386.

Warang, P., Kedar, P., Ghosh, K., \& Colah, R. B. (2012). Hereditary non-spherocytic hemolytic anemia and severe glucose phosphate isomerase deficiency in an Indian patient homozygous for the L487F mutation in the human GPI gene. International Journal of Hematology, 96, 263-267.
Whillier, S., Raftos, J. E., Sparrow, R. L., \& Kuchel, P. W. (2011). The effects of long-term storage of human red blood cells on the glutathione synthesis rate and steady-state concentration. Transfusion, 51(7), 1450-1459.

Zaidi, A. U., Kedar, P., Koduri, P. R., Goyette, G. W. J., Buck, S., Paglia, D. E., \& Ravindranath, Y. (2017). Glucose phosphate isomerase (GPI) tadikonda: Characterization of a novel Pro340Ser mutation. Pediatric Hematology and Oncology, 34, 449-454. 